\title{
MAN_02 - Situational Strategic Planning of the Pharmacovigilance of Covid-19 Vaccine (ChAdOx1-S [Recombinant]) at Bio-Manguinhos/Fiocruz
}

Paulo Roberto Gomes Takey ${ }^{1 *}$; Patrícia Mouta Nunes de Oliveira ${ }^{1}$; Letícia Kegele Lignani ${ }^{1}$; Renata Saraiva Pedro'; Maria de Lourdes de Sousa Maia ${ }^{1}$.

${ }^{1}$ Fiocruz/Bio-Manguinhos.

Introduction: The World Health Organization declared the outbreak of covid-19 a Public Health Emergency of International Concern in January 2020 and a pandemic in March 2020. Since the beginning of this confrontation, Fiocruz has been being part of several national and international fronts, including the search for the vaccine, together with the Ministry of Health. Fiocruz main strategy is an agreement with the biopharmaceutical company AstraZeneca to produce the covid-19 vaccine (ChAdOx1-S [recombinant]), developed by the University of Oxford, at Bio-Manguinhos. Collaborating with these efforts, the Clinical Advisory Unit/Bio-Manguinhos/Fiocruz conducted a Situational Strategic Planning (SSP) in the Pharmacovigilance Unit in order to comply with vanguard adverse events following immunization (AEFI) surveillance activities.

Objective: Identify opportunities for improvement; Implement changes; and Assess changes.

Methodology: A descriptive study based on strategic situational planning of pharmacovigilance activities was conducted, in four steps, from analysis of data collected systematically and compiled into a matrix, in the second half of 2020. Current standard operations procedures, technology transfer Safety Data Exchange Agreement (SDEA), National Regulatory Agency Good Pharmacovigilance Practices resolution, and COVAX Vaccine Safety, Developing Countries Vaccine Manufactures Network Pharmacovigilance Working Groups besides, National Immunization Program Rapid Response committee recomendations were considered. The Explanatory Moment consisted of explaining reality and reflecting problems; The Normative Moment, the description of proposed interventions, responsible and participants of activities, execution time and necessary resources; The Strategic Moment, the adversities during the process of implementing the strategic planning; and The Tactical-Operational Moment, the execution and monitoring of actions that were intended to be implemented.

Results: We developed an up to date SDEA in alignment with AstraZeneca. We prepared a comprehensive Risk Management Plan which includes an stimulated passive pharmacovigilance process, in addition to a post-authorisation safety study, both aiming to find identified and potential risks, besides missing information, in alignment with Anvisa, and national and international working groups. We've been developing answers to frequently asked questions or new scientific and technical information in alignment with Communication Advisory Unit, Post-Marketing Customer Service Division, health professionals associations and civil society organizations. We implemented an electronic pharmacovigilance system to optimize the continuous assessment of the benefit-risk profile. We participate in courses and lectures as listeners or speakers of covid-19, covid-19 vaccine and diagnostic kits.

Conclusion: Breaking out paradigm and investing in innovative pharmacovigilance approaches is essential in face of current regulatory requirements and public opinion about covid-19 vaccines. Continuous dynamic thinking and fast proactivity in the detection, assessment, understanding, prevention and communication of AEFI certainly can interrupt rumors and raise confidence on the immunization program consolidated over decades.

Keywords: Covid-19 vaccine; Pharmacovigilance; Situational Strategic Planning 